ICNMM2017-5501

\title{
MODELING OF ELECTRO-KINETIC MOTION OF JANUS DROPLET
}

\author{
S. Doğan Öner \\ Microfluidics \& Lab-on-a-chip Research Group \\ Mechanical Engineering Department \\ I.D. Bilkent University \\ Ankara 06800 TURKEY \\ Email: dogan.oner@bilkent.edu.tr
}

\author{
Barbaros Çetin* \\ Microfluidics \& Lab-on-a-chip Research Group \\ Mechanical Engineering Department \\ i.D. Bilkent University \\ Ankara 06800 TURKEY \\ Email: barbaros.cetin@bilkent.edu.tr
}

\begin{abstract}
Electro-kinetic manipulation Janus particles and droplets has attracted attention in recent years due to their potential application in microfluidics. Due to the presence of two different zone on the surface of particles with different charge distribution, the motion of the Janus particles are quite different than the that of regular particles. Therefore; the fundamental understanding of this motion is the key element for the further development of the microfluidic systems with Janus particles. In present study, electro-kinetic motion of Janus droplets inside a micro-channel is modeled using boundary element formulation. $2 D$ formulation is verified against the reported experimental data in the literature. Results show that the $2 D$ boundary element formulation is successful for the prediction of the electrophoretic velocity of the Janus droplets. The current formulation has a potential to model non-spherical particles and to study particle-particle and particle-wall interactions.
\end{abstract}

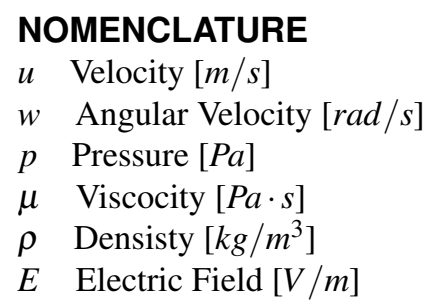

${ }^{*}$ Address all correspondence to this author. $\phi \quad$ Electric Potential [V]

$\zeta_{w} \quad$ Zeta Potential of channel wall $[\mathrm{mV}]$

$\zeta_{p} \quad$ Zeta Potential of particle $[\mathrm{mV}]$

$\varepsilon_{m}$ Permittivity of suspension medium $[\mathrm{C} / \mathrm{Vm}]$

$\theta \quad$ Surface covarage angle $\left[{ }^{\circ}\right]$

\section{INTRODUCTION}

Janus particles/droplets basically have two different polarizable surface when DC electric field is applied. Therefore; the induced charges have opposite signs on the particle surface. One surface facing electric field is negatively charged, the remaining surface is positively charged. As a result, dipolar electric doublelayer (EDL) causes reversed around the particle which results in vortices around the Janus particle. These vortices directly influence the motion of Janus particle in a micro-channel which is quite different than the electrophoretic motion of the regular particles with a uniform polarizable surface. This aspect opens up new possibilities for different applications. These particles can be utilized for self-assembling into simple or complex architecture [1], and/or utilized for mixing which is quite a challenge for many microfluidic applications. Moreover, utilizing the vortices, the motion of the particle can be controlled which would allow the differentiation of the Janus particles based on surface charge and size [2] and design of a microfluidic valve [3].

Electro-kinetic motion of Janus particles such as metal cylinder or sphere in liquid has been studied intensively for many 
years. Analytical expressions for electrophoresis of metallodielectric Janus particle in 2D was derived [4] and experimentally verified [5]. It was observed that Janus particles have tendency to move towards its dielectric side in AC electric field since electroosmotic flow on the metallic side of Janus particle. The numerical method is developed for the calculating the induced zeta potential on an arbitrary shape of totally polarizable particle [6,7]. Electro-kinetic motion of Janus particles with different polarizabilities in microchannel except for metallodielectric particles are studied both theoretically and experimentally $[3,8,9]$. Janus droplets/capsules also attracted attention for recent years, due to the flexibility of their fabrication and ability to generate fully polarizable surface together with dielectric surface. For the electro-kinetic Janus droplets, both theoretical and experimental studies are limited in comparison with Janus particles. In a recent study, the vortices around Janus droplets under the external DC electric field is numerically and experimentally demonstrated [2]. Due to the flexibility in the fabrication Janus droplets with different size and the ratio of the coverage of the polarizable surface can be controlled. The effect of the particle size, coverage ratio of the polarizable surface and aqueous solution on electrophoretic velocity and the intensity of the induced vortices has been studied [10,11].

Although there have been an increasing effort on the understanding of the electrophoretic motion of Janus particles, the analytical and numerical models are only for simple microfluidic settings with single spherical particles. Recently, our group developed a boundary element formulation for the modeling of dielectrophoretic particle motion more specifically the effect of particle-particle and particle-wall interaction [12]. Boundary element technique is a very powerful tool based on boundary discretization for problems whose governing equations are linear and offers a unique advantage for simulations with finite-sized particles since it does not require any re-meshing of the domain. As the particle moves in a micro-channel, the mesh elements on the particles translate and rotate. In this study, our boundary element formulation is extended to study the electrophoretic motion of Janus droplet. The results of the $2 \mathrm{D}$ model is verified against the recent experimental data for a singly Janus droplet. The current computational model has a potential to study the electrophoretic motion non-spherical particles, particle-particle and particle-wall interactions with high accuracy.

\section{THEORY}

Janus droplet have opposite sign of induced surface charges under the DC electric field. Due to the opposite sign of zeta potential , vortices are generated around the Janus droplet. Therefore; nature of Janus droplet results in multi-physic problem. Electric field and flow field of the system are taken into consideration in order to simulate electro-kinetic motion of the Janus droplet. In this study, Janus droplet that is non-conducting sim-

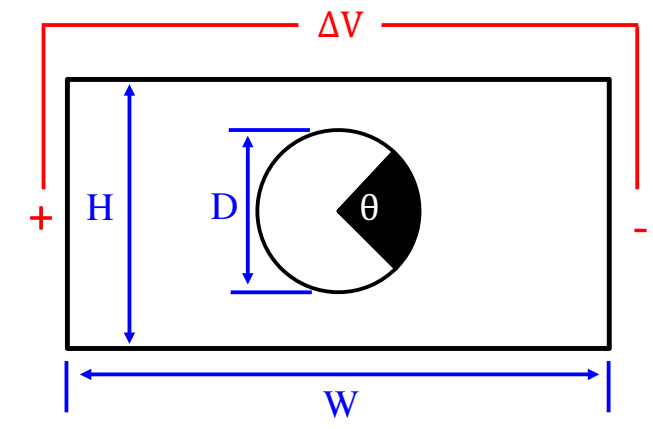

FIGURE 1. SCHEMATICS OF THE PROBLEM

ply considered as a solid particle because of the presence of impurities at the liquid-liquid interface [13] and the motion inside droplet is neglected. Change in motion of the Janus droplet under the DC electric field with different surface coverage, dimensions and aqueous solution is main focus in this study.

\section{Electric Field}

Electrical potential $\phi$ of Janus droplet under the DC electric field in aqueous solution can be determined by solving Laplace's equation Eq.1.

$$
\nabla^{2} \phi=0
$$

For the boundary conditions, electric potential difference is applied $(\Delta V)$ at the channel inlet and outlet. For the channel wall, electric insulation is implemented. For the Janus droplet, it is considered as a non-conducting droplet in Fig. 1

\section{Flow Field}

Considering the low Reynolds number nature of flow, Stokes equation Eq. 3 and contuinty equation Eq. 2 can be solved to determine the flow field.

$$
\begin{gathered}
\nabla \cdot \mathbf{u}=0 \\
-\nabla p+\mu \nabla^{2} \mathbf{u}=0
\end{gathered}
$$

For the boundary condition, pressure difference between inlet and exit of the channel is taken zero. Using thin-electric double layer (EDL) assumption, the slip velocity is applied at the channel wall :

$$
u_{s l i p, \|}=-\frac{\varepsilon_{m} \zeta_{w}}{\mu}(\nabla \phi)_{\|}
$$


where $\zeta_{w}$ is the zeta potential of the channel wall. The same slip velocity is also defined on the particle surface by replacing the zeta potential with that of the particle.

Considering very thick EDL, electrophoretic velocity of particle in unbounded aqueous electrolyte solution is [14].

$$
U_{e p}=\frac{2}{3} \frac{\varepsilon_{m} \zeta_{p}}{\mu} \vec{E}
$$

\section{BOUNDARY ELEMENT FORMULATION}

Modeling of Janus Droplet of with BEM requires the solution of the electric field equation to obtain the electro-kinetics forces on the particle, and the solution of the Stoke's flow equation to obtain the hydrodynamic forces. The boundary element equation for Laplace Equation can be written as [12]:

$$
C(A) \phi(A)+\int_{S} q^{*}(A, P) \hat{\phi}(P) \mathrm{d} S=\int_{S} \phi^{*}(A, P) \hat{q}(P) \mathrm{d} S
$$

where $A$ is the source point and $P$ is the field point. In Eq. (6), $\hat{\phi}$ represents the electric potential, and $\hat{q}$ represents the corresponding normal flux on the boundary. $\phi^{*}$ and $q^{*}$ are the so called fundamental solutions of the Laplace equation. The constant $C(A)$ takes values depending on the position of the source point $A$ : If $A$ is inside the solution domain, $C(A)=1.0$, if $A$ is on a smooth boundary $C(A)=0.5$, if $A$ is outside the solution domain $C(A)=0$. Following the discretization, the integral equation becomes:

$$
\mathbf{H}^{\phi} \cdot \phi=\mathbf{G}^{u} \cdot \mathbf{q}
$$

Here, $\phi$ represents the column electric potential vector , q represents the corresponding fluxes at the boundary nodes and $\mathbf{H}^{\phi}$ and $\mathbf{G}^{\phi}$ are the system matrices constructed using the fundamental solutions of the Laplace equation. Through the imposition of proper boundary conditions, these quantities are pre-determined leading to a solvable system of linear equations. The solution of the equations lead to the determination of the the electric potential $(\phi)$ at each boundary node along with the normal flux. For the evaluation of the MST which lead to the $\mathbf{F}_{E K}$, the proper determination of $\mathbf{E}(=-\nabla \phi)$ is required.

The boundary element equation for Stoke's equations can be written as [15]:

$$
C_{i j}(A) u_{j}(A)+\int_{S} t_{i j}^{*}(A, P) u_{i}(P) \mathrm{d} S=\int_{S} u_{i j}^{*}(A, P) t_{i}(P) \mathrm{d} S
$$

where $u_{i}$ are the components of the velocity vector and $t_{i}$ are the components of the traction vector at the given point. $u_{i j}^{*}$ and $t_{i j}^{*}$ are the fundamental solutions of Stoke's equation. Similarly, Constants $C_{i j}(A)$ take values depending on the position of the point $A$ : If $A$ is inside the solution domain, $C_{i j}(A)=\delta_{i j}$, if $A$ is on a smooth boundary $C_{i j}(A)=\delta_{i j} / 2$ and if $A$ is outside the solution domain $C_{i j}(A)=0$. The resulting system of equations are very similar to those of Eq. (7):

$$
\mathbf{H}^{u} \cdot \mathbf{u}=\mathbf{G}^{u} \cdot \mathbf{t}
$$

where $\mathbf{u}$ is a velocity field vector at each boundary node and $\mathbf{t}$ is a traction vector at the same boundary nodes. The system matrices, $\mathbf{H}^{u}$ and $\mathbf{G}^{u}$ are constructed using the fundamental solutions of the Stoke's equation. Boundary conditions are implemented, thus corresponding velocities and tractions for each node are achieved. After that, assuming rigid particles, the motion characteristic is $2 \mathrm{D}$ rigid-body motion which means each boundary point has a velocity given by [12]:

$$
u_{i}=u_{i}^{b}+\omega r \hat{t}_{i}
$$

$u_{i}$ are the components of the velocity vector at a node on the boundary of the particle, $u_{i}^{b}$ are the components of the velocity vector at the selected center of the rigid particle, $\omega$ is the angular velocity of the particle, $r$ is the distance between the selected center and the boundary node and $\hat{t}$ are the components of the unit-perpendicular vector to the vector drawn from the center to the node. Center velocity of particle is determined by imposing rigid-body motion condition.

\section{RESULT AND DISCUSSION}

To mimic the experimental setting used in [2], a channel with a width of $150 \mu \mathrm{m}$ and a length of $1000 \mu \mathrm{m}$ is used. A droplet with a diameter of $75 \mu \mathrm{m}$ is placed at the inlet from the centerline. The angular coverage of the polarizable surface $(\theta)$ is taken from the study of $\mathrm{Li}$ and $\mathrm{Li}$ [2] by digitizing the images. The zeta potential of the channel wall, non-conducting surface of the particle and the polarizable surface of the particle is set to $\zeta_{w}=-60 \mathrm{mV}$, $\zeta_{p+}=60 \mathrm{mV}$ and $\zeta_{p-}=-40 \mathrm{mV}$. The applied electric field is taken in the range of $50-100 \mathrm{~V} / \mathrm{cm}$. In BEM analysis, unless otherwise stated, 4 boundary element per micrometer is used for discretizing the channel walls, and 5 boundary elements per micrometer is used for discretizing the particle boundary. Constant elements are implemented. Eulers method is implemented for time integration. The convergence of the particle trajectory is achieved by the time step of $\mathrm{dt}=0.001 \mathrm{~s}$ for all the simulations. The convergence criteria are set as the deviation of 0.01 . Numerical integrations are performed using Gauss-quadrature with 24 points. Singular integrals are evaluated analytically. 

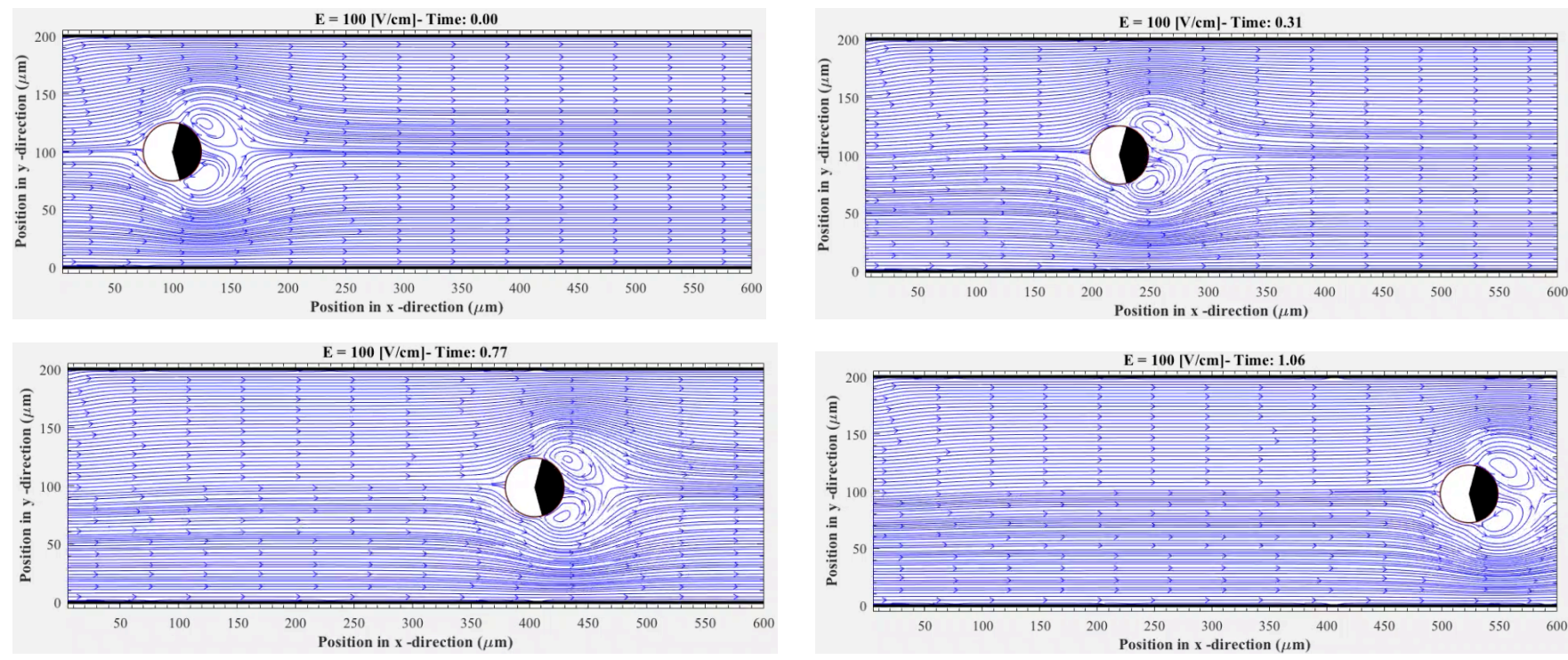

FIGURE 2. ELECTROPHORETIC MOTION OF A JANUS DROPLET

Figure 2 shows the electrophoretic of the oil droplet $(\theta=$ $160^{\circ}$ ) for an electric field of $100 \mathrm{~V} / \mathrm{cm}$, channel with a width of $200 \mu \mathrm{m}$ and a length of $600 \mu \mathrm{m}$ is used. Due to the imbalanced slip-velocities in opposed directions, vortices are generated on the polarizable surface of the particle. The particle moves along the centerline of the micro-channel. Although it has been shown in here, the size of the vortices grows with the applied electric field.

To further explore the effect of different parameters on the electrophoretic velocity of the particle, several different cases are simulated:

Case-A: $\mathrm{D}=75 \mu \mathrm{m}, \theta=160^{\circ}, \zeta_{w}=-60 \mathrm{mV}$
Case-B: $\mathrm{D}=75 \mu \mathrm{m}, \theta=100^{\circ}, \zeta_{w}=-60 \mathrm{mV}$
Case-C: $\mathrm{D}=45 \mu \mathrm{m}, \theta=160^{\circ}, \zeta_{w}=-60 \mathrm{mV}$
Case-D: $\mathrm{D}=75 \mu \mathrm{m}, \theta=160^{\circ}, \zeta_{w}=-20 \mathrm{mV}$

The results are shown in Fig. 3. The theoretical value for a regular particle with a thin EDL assumption is also given the figure with a black solid line. As seen from the figure, our BE formulation can predict the general trend of the behavior. Form quantity point of view, the electrophoretic velocity of the oil droplet is slightly underestimated. The main reason for that is the 2D modeling of the actual 3D problem. Strictly speaking, 2D modeling represents the flow of a cylinder. However, in real situation particle is a sphere. The resistant of the cylinder to the flow would be higher than that of the sphere. This is the reason, 2D model predicts a lower velocity than that of the experiments. Referring to the Fig. 3-(A) and (B), the electrophoretic velocity increases with the coverage area of the polarizable surface, and the size. Furthermore, aqueous solution dramatically effects the electrophoretic velocity. Fig. 3-(C) clearly shows that increase in zeta potential of wall of channel decrease the electrophoretic velocity of droplet $n$ Fig. 3-(C).

\section{CONCLUSION}

In this paper, the electro-kinetic motion of an electrically induced droplet is simulated with BE formulation. Developed $\mathrm{BE}$ formulation and recent experimental data are compared. Although there is problem with 2D modelling of actual 3D model, the simulated results make a good agreement with experimental results. Also, it is understood that electrophoretic velocity of Janus droplet in microchannel depends on polarizable surfaces, dimensions and aqueous solution. 


\section{REFERENCES}

[1] Rozynek, Z., Mikkelsen, A., Dommersnes, P., and Fossum, J. O., 2014. "Electroformation of janus and patchy capsules". Nature Communications, 5, 05, pp. 3945 EP -.

[2] Li, M., and Li, D., 2015. "Vortices around janus droplets under externally applied electrical field". Microfluid Nanofluid, 20(79).

[3] Zhang, F., and Li, D., 2013. "Induced-charge electroosmotic flow around dielectric particles in uniform electric field". J. Colloid Interf. Sci., 410, pp. 102-110.

[4] Squires, T. M., and Bazant, M., 2004. "Induced-charge electro-osmosis". J. Fluid Mech., 509, pp. 217-252.

[5] Gangwal, S., Cayre, O. J., Bazant, M. Z., and Velev, O., 2008. "Induced-charge electrophoresis of metallodielectric particles". Phys. Rev. Lett., 100(058302).

[6] Wu, Z., Gao, Y., and Li, D., 2009. "Electrophoretic motion of ideally polarizable particle in microchannel". Electrophoresis, 30, pp. 773-781.

[7] Daghighi, Y., Gao, Y., and Li, D., 2011. “3d numerical study of induced-charge electrokinetic motion of heterogeneous particle in a microchannel". Electrochim. Acta, 56, pp. 4254-4262.

[8] Zhang, F., and Li, D., 2014. "A novel particle separation method based on induced-charge electro-osmotic flow and polarizability of dielectric particles". Electrophoresis, 35, pp. 2922-2929.

[9] Zhang, F., and Li, D., 2014. "Separation of dielectric janus particles based on polarizability- dependent induced-charge electroosmotic flow". J. Colloid Interf. Sci., 448, pp. 297305.

[10] Li, M., and Li, D., 2016. "Fabrication and electrokinetic motion of electrically anisotropic janus droplets in microchannels". Electrophoresis, 00(1-9).

[11] Li, M., and Li, D., 2017. "Electrokinetic motion of an electrically induced janus droplet in microchannels". Microfluidics and Nanofluidics, 21(2), p. 16.

[12] Cetin, B., Oner, S. D., and Baranoglu, B., 2017. "Modeling of dielectrophoretic particle motion: Point particle versus finited-sized particle". Electrophoresis, 00, pp. 1-12.

[13] Baygents, J. C., and Saville, D. A., 1991. "Electrophoresis of drops and bubbles". J. Chem. Soc., Faraday Trans., 87, pp. 1883-1898.

[14] Li, D., 2004. Electrokinetics in Microfluidics. Elsevier Academic Press, pp. 1-5.

[15] Karakaya, Z., Baranoglu, B., Cetin, B., and Yazici, A., 2015. "A parallel boundary element formulation for tracking multiple particle trajectories in Stoke's flow for microfluidic applications". CMES-Computer Modeling in Engineering and Science, 104, pp. 227-249.

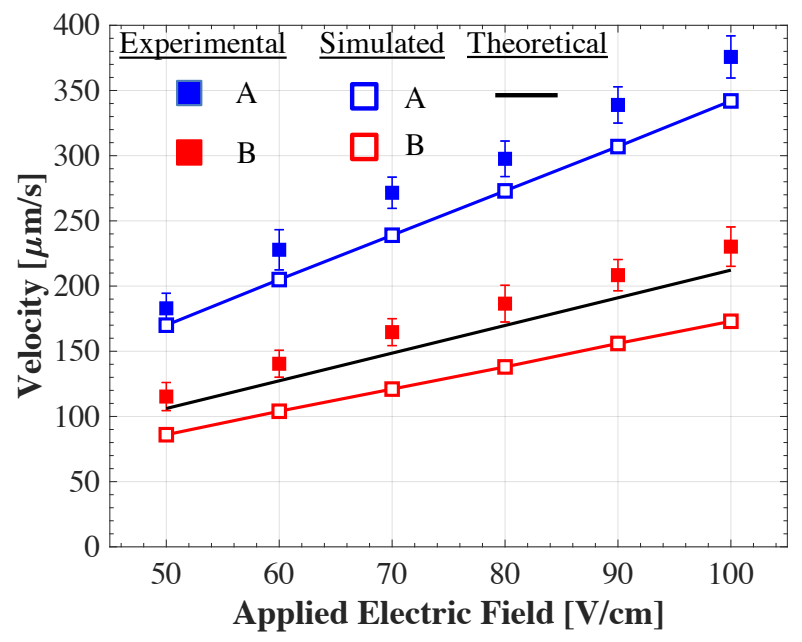

(A)

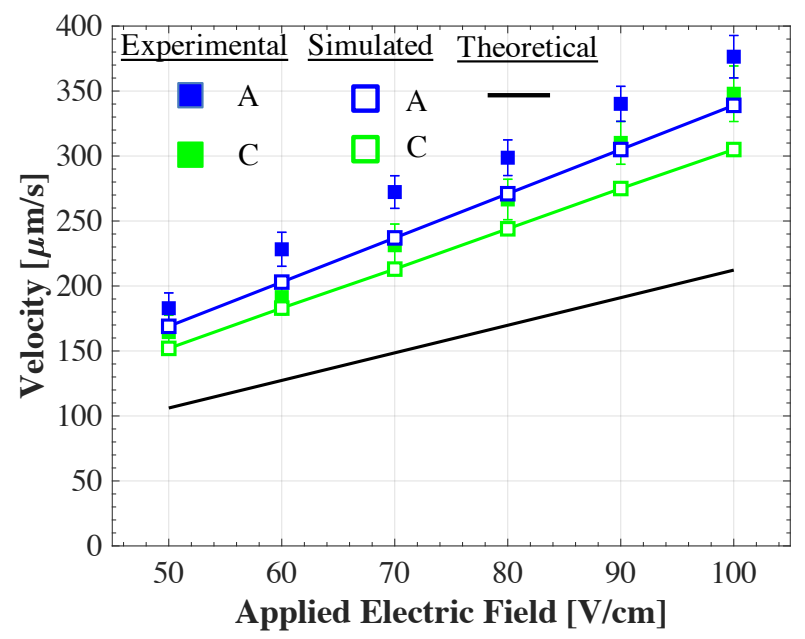

(B)

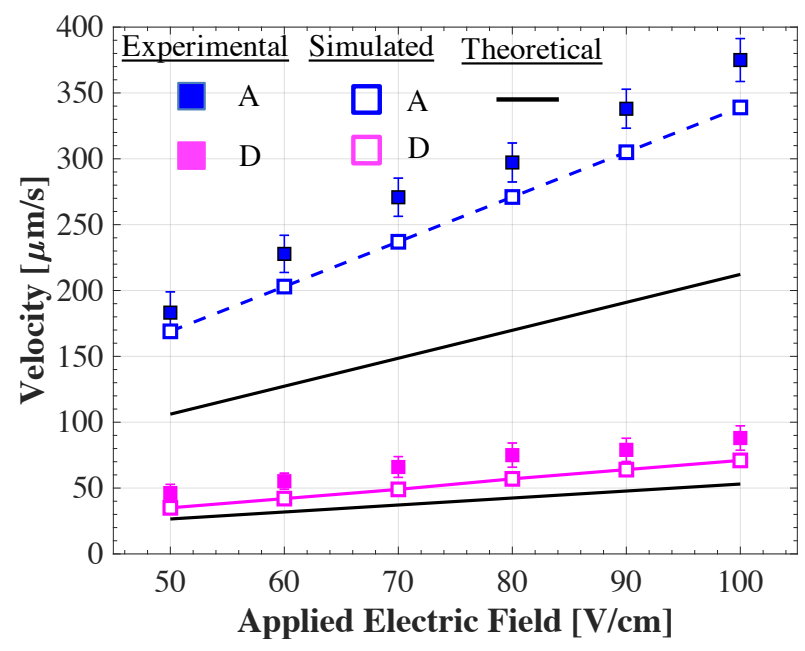

(C)

FIGURE 3. ELECTROPHORETIC VELOCITY 